\title{
Through the looking glass: counter-mirror activation following incompatible sensorimotor learning
}

Caroline Catmur* ${ }^{*}$, Helge Gillmeister ${ }^{1,2}$, Geoffrey Bird ${ }^{1}$, Roman Liepelt ${ }^{3}$, Marcel Brass ${ }^{3,4,5}$ \& Cecilia

$$
\text { Heyes }^{1}
$$

${ }^{1}$ Dept of Psychology, University College London, London WC1H OAP, UK.

${ }^{2}$ Dept of Psychology, City University, London EC1V OHB, UK.

${ }^{3}$ Dept of Cognitive Neurology, Max Planck Institute for Human Cognitive and Brain Sciences, Stephanstr. 1A, 04103 Leipzig, Germany.

${ }^{4}$ Dept of Experimental Psychology, Ghent University, Henri Dunantlaan 2, B-9000 Gent, Belgium.

${ }^{5}$ Ghent Institute for Functional and Metabolic Imaging, Ghent University Hospital, De Pintelaan 185, B-9000 Gent, Belgium.

*Corresponding Author: email c.catmur@ucl.ac.uk / phone +44 2076795379 / fax +44 2074364276

Running Title: Sensorimotor learning in the mirror system

Keywords: mirror neurons; imitation; action observation; experience; fMRI

Pages: 34

Figures: 3

Tables: 1

Text: 6,656 words including References and Figure Legends

Abstract: 207 words 
Introduction: 496 words

Submitted: $9^{\text {th }}$ April 2008; re-submitted: $3^{\text {rd }}$ June 2008; final version submitted July 2008

Abstract

The mirror system, comprising cortical areas that allow the actions of others to be represented in the observer's own motor system, is thought to be crucial for the development of social cognition in humans. Despite the importance of the human mirror system, little is known about its origins. We investigated the role of sensorimotor experience in the development of the mirror system. Functional Magnetic Resonance Imaging (fMRI) was used to measure neural responses to observed hand and foot actions following one of two types of training. During training, participants in the Compatible (control) group made mirror responses to observed actions (hand responses were made to hand stimuli and foot responses to foot stimuli), while the Incompatible group made countermirror responses (hand to foot and foot to hand). Comparison of these groups revealed that after training to respond in a counter-mirror fashion, the relative action observation properties of the mirror system were reversed: areas which showed greater responses to observation of hand actions in the Compatible group responded more strongly to observation of foot actions in the Incompatible group. These results suggest that, rather than being innate, or the product of unimodal visual or motor experience, the mirror properties of the mirror system are acquired through sensorimotor learning. 

Introduction

The "mirror system", first discovered in the macaque (di Pellegrino et al., 1992), is active both when we perform an action ourselves, and when we observe another person performing the same action. fMRI has revealed mirror activity of this kind in bilateral premotor and inferior parietal cortices (e.g. lacoboni et al., 1999;Vogt et al., 2007), areas considered homologous with macaque mirror neuron locations. Further support for the existence of a human mirror system stems from transcranial magnetic stimulation (Fadiga et al., 1995;Strafella and Paus, 2000;Catmur et al., 2007) and behavioural (Sturmer et al., 2000;Brass et al., 2001a) studies demonstrating that observation of an action automatically activates a corresponding motor representation in the observer.

It has been suggested that the mirror system plays a crucial role in social cognition (Gallese, 2007), action understanding (Rizzolatti and Craighero, 2004) and the evolution and development of language (Rizzolatti and Arbib, 1998; Theoret and Pascual-Leone, 2002;Arbib, 2005). Despite its importance, almost nothing is known about the mirror system's phylogenetic and ontogenetic origins. It is frequently implied that natural selection has endowed the system with its mirror properties (e.g. Gallese and Goldman, 1998). Alternative theories suggest that the properties of the mirror system are acquired through lifelong sensorimotor experience in which the individual simultaneously observes and executes the same action (Heyes, 2001;Hommel et al., 2001;Keysers and Perrett, 2004). This hypothesis suggests that, if an infant were unable to see her own actions, and grew up in an environment where mirrors and imitating adults were replaced by systems that showed counter-mirror actions (e.g. foot movements when she moved her hands), then she would develop a counter-mirror system. When she observed an action, those areas that normally develop into the mirror system would show activation associated with execution of a different action. 
Rather than attempting this cruel experiment, the present study tested the sensorimotor learning hypothesis with adults. We used fMRI to compare the neural activity evoked by action observation after compatible and incompatible sensorimotor training. Before scanning, participants in the Compatible control group were trained to raise their hand whenever they saw a raised hand action, and raise their foot whenever they saw a raised foot. The Incompatible group raised their hand whenever they saw a raised foot, and raised their foot whenever they saw a raised hand (see also Gillmeister et al., 2008). If development of the mirror system depends on sensorimotor experience, one would expect the Incompatible group to show "counter-mirror" activity in the mirror system during subsequent action observation, possibly in a somatotopic fashion (Buccino et al., 2001). If, however, effector somatotopy is not present, then, based on studies of activity in premotor cortex during action execution (Kollias et al., 2001), one would expect the Compatible group's mirror system to be more active during observation of hand than foot actions. In contrast, because observation of foot actions has become associated with performance of hand actions, the Incompatible group's mirror system should be more active during observation of foot than hand actions. 
Materials and Methods

Participants

Twenty right-handed participants (11 male, 9 female; mean age 25.4 years; see Gillmeister et al., 2008) were randomly assigned in equal numbers to either the Compatible or Incompatible training group. All had normal or corrected-to-normal vision and were naïve with respect to the purpose of the experiment. Participants gave their written informed consent to participate in the study, which was approved by the ethics committee of the University of Leipzig and conducted in accordance with the ethical standards laid down in the 1964 Declaration of Helsinki.

Stimuli

Training

During the training phase, each trial consisted of a warning and an imperative stimulus. Warning stimuli consisted either of a compound image of a hand and a foot side by side in a resting position (compound stimulus), or of a single image of either a hand or a foot in a resting position presented in the centre of the screen (single stimulus). The imperative stimulus consisted of either a hand or a foot in a raised position. For compound stimuli, the other effector remained in the resting position.

In order to encourage attention to the stimuli during training (and to decrease the risk of repetition suppression during scanning sessions) a number of different hand and foot stimuli were used. Images of hand and foot postures were recorded from four different models (one male, three female), at two different viewpoints (from the side and from the side with a heightened elevation) and at two sizes. When raised, the hand was flexed at the wrist by an angle of between $40^{\circ}$ and $65^{\circ}$, 
and the foot was flexed at the ankle by an angle of between $16^{\circ}$ and $51^{\circ}$. When compound stimuli were presented, hand and foot images from the same model were always presented together. See Figure 1 for timelines depicting stimuli and procedures.

Functional Imaging Session

Stimuli were the same as for the training sessions, with the exception that only single stimuli were presented.

Insert Figure 1 about here

Procedure

Training

Training was completed outside the scanner. Participants sat at a viewing distance of approximately $60 \mathrm{~cm}$ in front of the stimulus presentation screen. The participant's right forearm lay prone, in a horizontal position, away from his/her body, supported by an armrest from the elbow to the wrist. The lower right leg was slightly stretched away from the body in the same direction as the arm, with the foot resting firmly on the floor. Participants made hand / foot movement responses by abducting the hand / foot at the wrist / ankle such that the front of the hand / foot moved upwards. After making each response, participants returned their hand or foot to the resting position.

Each trial began with the presentation of the warning stimulus. The warning stimulus was shown for a variable duration between 800 and $1280 \mathrm{~ms}$ before it was replaced by the imperative stimulus 
which was shown for 640ms. Each trial therefore depicted either a hand or foot being raised from a resting position either alone (single stimulus), or while the other effector remained at rest (compound stimulus). The compound stimuli introduced uncertainty as to which response would be required, thus response selection could not take place until the onset of the imperative stimulus. This ensured that new associations were formed between dynamic, rather than static, properties of the stimuli, and the responses. Participants were instructed to respond to the imperative stimulus as quickly as they could, without making errors, by raising their hand or their foot. Participants in the Compatible training group were instructed to raise their hand as soon as possible if they saw a raised hand, and to raise their foot as soon as possible if they saw a raised foot. Participants in the Incompatible training group were instructed to raise their hand as soon as possible if they saw a raised foot, and to raise their foot as soon as possible if they saw a raised hand.

Onset of hand and foot responses was measured by recording the electromyogram (EMG) from the flexor carpi radialis muscle of the right forearm and the tibialis anterior muscle of the lower right leg. The response time (RT) interval began with the onset of the imperative stimulus, and ended with EMG onset (see Heyes et al., 2005, for details).

Training was conducted over the course of three consecutive days. On each day, the training session lasted approximately 45 minutes. Each session consisted of 384 trials, divided into six blocks by short breaks. There were 16 trial types, defined by effector moved (hand or foot), stimulus type (compound or single stimulus), viewpoint (side or elevated), and size (large or smaller). These were equiprobable and randomly intermixed within each training session. Compound stimuli were presented equally often with the hand on the left and the foot on the right of the screen, and vice versa. The location of the hand with respect to the foot was always the same for warning and imperative stimuli within each trial. Each session was preceded by 12 practice training trials in order to ensure that participants were performing the task correctly. 
Before the second and third training sessions, RTs obtained over the course of the previous training session(s) were shown to the participant in order to encourage maximal performance. Performance improvement (response speed and / or error rate) over the course of training was further encouraged by offering financial incentives for better performance and financial penalties for worse performance relative to the previous session.

Each training session was preceded by a short execution practice session (10 trials) in which participants practised performing the actions they would make in the Execution Task during the functional imaging session. To discourage movement planning during the Observation Task (which always preceded the Execution Task during the imaging session), participants were not informed that they would perform these actions in the scanner, but instead were told that the actions were performed to "warm up" their muscles for the training sessions. During these practice sessions the experimenter read out a simple instruction for an action, which the participant then performed repeatedly for ten seconds. The actions comprised five movements of the hand (rotate hand clockwise, move hand left and right, make a fist, spread the fingers apart, wriggle the fingers), and five equivalent foot movements (rotate foot clockwise, move foot left and right, roll up the toes, spread the toes apart, wriggle the toes). This range of actions was chosen to be different from those used in the Observation Task and during training, because their purpose was to localise cortical areas involved in the performance of hand and foot actions in general. For the hand actions, the lower arm was placed from elbow to wrist on the arm rest, allowing free movement of the hand. For the foot actions, the leg was placed on a chair from knee to ankle, allowing free movement of the foot. All actions pertaining to the hand, and all actions pertaining to the foot, were performed en bloc. The order of blocks, and the order of actions within each block, was determined randomly. The experimenter monitored that the participant had understood the instruction and reminded participants, if necessary, to restrict movement to the hand / foot. 


\section{Functional Imaging Session}

The functional imaging session was completed twenty-four hours after the third training session and comprised the Observation Task and the Execution Task. The Observation Task always preceded the Execution Task. The participant lay in a supine position inside the scanner. Their right arm was placed on a cushion from elbow to wrist, and their right lower leg was placed on a cushion from knee to ankle, allowing free movement of hand and foot during the Execution Task. They were instructed that this was to check they were not moving during the Observation Task, in order to avoid providing any information about the later Execution Task and possibly prompting movement planning during the Observation Task. 


\section{Observation Task}

During the Observation Task participants observed hand and foot actions without responding. Participants observed a total of 128 hand and 128 foot actions, interspersed with six catch trials (three incomplete hand lifting and three incomplete foot lifting actions) and 36 null events (blank screen replaced stimulus presentation). Observation trials followed the structure of training trials (warning stimulus presented for between $800 \mathrm{~ms}$ and $1280 \mathrm{~ms}$, followed by the action stimulus for $640 \mathrm{~ms}$ ). Each trial was preceded by a black screen of variable duration (mean: 3920ms, range: $2580 \mathrm{~ms}$ to $6060 \mathrm{~ms}$ ). Each of the eight stimulus types (defined by factorial combination of the effector moved, viewpoint and size variables) was shown an equal number of times and in random order. In order to encourage attention to the stimuli, participants were asked to observe closely and report anything unusual (the six catch trials) at the end of the session.

\section{Execution Task}

During the Execution Task participants made hand and foot actions in response to written instructions. Participants performed the ten actions that they had practised at the start of each training session. Each trial was preceded by a blank screen of variable duration (mean: $2750 \mathrm{~ms}$, range: $2 s$ to $5 s)$. Written instructions then detailed the action to be executed (3s), followed by the word START (750ms). The participant then performed the action repeatedly (10s), until the word STOP appeared on the screen (750ms). Actions were performed in a random order. Each action was performed twice during the task. Rest blocks (of $18 \mathrm{~s}$ duration) were interspersed with action trials.

\section{Image Acquisition}

fMRI data were acquired with a T2* echoplanar sequence using BOLD (blood oxygenation level dependent) contrast on a Siemens Trio 3 Tesla system. Each functional brain volume comprised 24 slices of $5 \mathrm{~mm}$ thickness (1mm spacing), TE 30ms, TR $2 \mathrm{~s}$. The functional data were acquired in two sessions (one session for each task); the first six volumes of each 
session were discarded to allow for T1 equilibration effects. Stimulus presentation began after the sixth volume. A total of 1266 full-brain volumes for each participant were acquired over the two sessions.

\section{Data Analysis}

Data were analyzed using SPM2 (Wellcome Department of Imaging Neuroscience, London, UK) (Friston et al., 1995). Images were realigned and "unwarped" (corrected for interactions between movements and field inhomogeneities) (Andersson et al., 2001), normalised to a standard EPI template, resampled to a resolution of $3 \times 3 \times 3 \mathrm{~mm}$, and smoothed with a threedimensional Gaussian kernel with full-width half maximum of $6 \mathrm{~mm}$. In addition, a high-pass temporal filtering with a cut-off of 128 secs was applied in order to exclude low-frequency artefacts. After pre-processing, statistical analysis was carried out using the general linear model (GLM; Friston et al., 1995). Each observation trial was modelled by a standard haemodynamic response function. Execution trials (from the onset of action instructions to cessation of movement) were modelled by convolving a box-car function with a standard haemodynamic response function. These observation-related and execution-related effects were modelled within a single mixed-design GLM, allowing separation of the influences of these factors on neural activity (see Laurienti et al., 2003). To allow inferences at the population level, a second-level random effects analysis was performed using ANOVA on contrast images of the different conditions for each individual subject.

As an initial analysis, we compared action execution between the two groups to verify that there were no differences in the groups' activation patterns during the Execution Task. This SPM was thresholded at $\mathrm{p}<0.05$ corrected for whole brain volume.

The second analysis identified mirror system areas. A conjunction (null) was performed on the contrast images comparing action observation (of both hands and feet) to baseline, and action execution (of both hands and feet) to baseline, in the Compatible training group. We 
restricted the conjunction to the Compatible training group in order to avoid any contamination of the classical activation pattern by incompatible training. The resulting SPM was thresholded at $\mathrm{p}<0.05$ corrected for whole brain volume.

We next wanted to test whether we could identify, within mirror system areas, a somatotopic representation of the observed effector in the Compatible training group. While such a somatotopy has been suggested by earlier imaging work (Buccino et al., 2001; Wheaton et al., 2004), these experiments did not include an action execution condition, and therefore it is unclear whether the areas identified were truly "mirror" (active for both execution and observation of actions). Additionally, recent studies have not shown clear differences at the group level between cortical areas responding to observation of hand and foot actions (AzizZadeh et al., 2006;Gazzola et al., 2007). We therefore compared activation for the observation of hand actions with observation of foot actions and vice versa, in the mirror areas defined by our initial conjunction analysis. The resulting SPM was thresholded at $\mathrm{p}<$ 0.05 corrected for the search volume.

The fourth analysis addressed our main experimental question, with regard to which we were primarily interested in the interaction of training group and observed effector representation in the mirror system in general. In particular we wanted to test whether the activation strength for observed hand and foot actions was influenced by incompatible training. In order to address this question we used a voxel of interest approach. The peak voxels in the mirror areas as defined by our initial conjunction analysis and monkey neurophysiology (bilateral premotor and inferior parietal cortices) were selected and parameter estimates for activity during action observation in both groups were extracted. These data were entered into $2 \times 2$ mixed model ANOVAs at each of these voxels with a within-subjects factor of observed action (hand or foot) and a between-subjects factor of group (Compatible or Incompatible training). 
In order to verify whether these areas of the mirror system are dominant for the observation and execution of hand actions over foot actions, we compared the location of these peak voxels with those selected based on the Compatible training group's responses to hand actions only. As a fifth analysis, we performed a conjunction of observed and executed hand actions, in the Compatible training group, within the mirror system areas defined above. The SPM from this conjunction was thresholded at $\mathrm{p}<0.05$ corrected for the search volume. Finally, a similar conjunction was also performed for observed and executed foot actions. 
Results

The initial analysis of the Execution Task data revealed no differences between the groups for action execution.

In accordance with previous studies, the second, conjunction analysis, of action execution and action observation for both actions in the Compatible training group, revealed a number of brain regions (Table 1) including bilateral premotor cortex and inferior parietal cortex (Figure 2). As outlined above, we restricted the subsequent analyses to these areas, because, based on single-cell recording in the macaque, they are thought to form the core mirror system (Rizzolatti and Craighero, 2004).

Insert Table 1 about here

Insert Figure 2 about here

We next investigated whether action observation somatotopy (Buccino et al., 2001) was present in the mirror system in the Compatible training group. That is, we asked whether there are areas in the mirror system that are organised somatotopically by observed effector, with certain regions responding preferentially to the observation of hand actions and others to the observation of foot actions. We did not find any voxels in the mirror system, as defined by the conjunction analysis above, that were significantly more active during observation of hand than foot actions, or vice 
versa. This was also the case when the statistical thresholds, for both the conjunction analysis and the analyses of somatotopy, were lowered to $p<0.001$, uncorrected.

Since we did not find a somatotopic organisation of the observed effector in the mirror system, for the subsequent, fourth analysis we investigated activation in the mirror system in general, as defined by the conjunction analysis. The prediction that the Incompatible training group would show counter-mirror activity during action observation was investigated by extracting the data from the peak voxels in these classical mirror system areas (bilateral premotor and inferior parietal cortices). We tested our prediction by computing the two-way interaction between the observed action effector (hand or foot) and training group (Compatible or Incompatible), using mixed-model ANOVAs. This interaction was significant in all four mirror system areas (left premotor cortex, $F_{1,18}=$ $6.839, p=0.018$; right premotor cortex, $F_{1,18}=10.618, p=0.004$; left inferior parietal cortex, $F_{1,18}=$ 7.706, $p=0.012$; right inferior parietal cortex, $F_{1,18}=7.603, p=0.013$; see Figure 3 ). As can be seen from Figure 3, all regions showed a dominance for observation of hand over foot actions in the Compatible training group which was reversed to a foot dominance in the Incompatible training group.

Insert Figure 3 about here

The dominance in the Compatible training group for observation of hand over foot actions is consistent with other findings (e.g. Kollias et al., 2001) suggesting greater representation of hand than of foot actions in these areas. This suggests that a conjunction analysis based on observation and execution of hand actions alone in the Compatible training group should identify peak voxels in similar locations to those identified across both effectors by the previous analysis. Indeed, this 
further conjunction analysis showed that the peak voxels in right premotor and inferior parietal cortex were in the same locations, while the remaining two peaks were within $5 \mathrm{~mm}$ of the original voxels (see Table 1). These two additional voxels showed the same pattern of dominance for observation of hand over foot actions in the Compatible training group, reversed to a foot dominance in the Incompatible training group. As a final analysis, we performed a conjunction analysis based on the observation and execution of foot actions alone in the Compatible training group. However, as a result of the hand dominance of these areas, the foot analysis showed a mostly similar pattern of results to the hand analysis. That is, while the peak voxels in the foot analysis are responsive to the observation and execution of foot movements, they are still more responsive to the observation of hand movements in the Compatible group.

Discussion

Mirror system areas were identified in this experiment as those active during both action observation and action execution. These showed a high degree of overlap with those reported in previous studies (lacoboni, 2005). We will discuss our findings relating to effector somatotopy in these mirror areas, before moving on to the effects of sensorimotor learning on the mirror system.

Within the mirror system, we did not find any areas that were significantly more active during the observation of hand than of foot actions, or vice versa. In this respect our results are in accordance with those of Aziz-Zadeh et al. (2006) who did not find a region of premotor cortex specific to observation of foot actions compared to observation of mouth or hand actions. These results differ, however, from those of Buccino et al. (2001) who reported somatotopic organisation of premotor and parietal cortex during observation of foot, hand and mouth actions. It is possible that the 
previously reported somatotopy is action, rather than effector, specific: in Buccino et al. (2001)'s study, the movement stimuli varied on the dimensions of both action and effector used (for instance, grasping with the hand versus kicking with the foot), and the contrasts used subtracted observation of the static effector from that of the moving stimuli, thus emphasising the action dimension. To our knowledge, the current brain-imaging literature on somatotopic organization of effector representations during action observation includes no studies in which effector and action type are not confounded. However, two recent behavioural studies have shown that movement priming can occur at the action-level instead of, or in addition to, the effector-level (Costantini et al., 2008; Leighton and Heyes, submitted). Thus, if somatotopic organisation reflects the action dimension, as also suggested by Schubotz and von Cramon (2003)'s concept of a "pragmatic body map" in premotor cortex, then the similarity of the actions performed by the two effectors in the present study, while providing a high level of experimental control, may have prevented the detection of somatotopically organised representations of effectors during action observation.

While other studies have compared neural responses during the performance of matching and nonmatching actions (Brass et al., 2001b;Alegre et al., 2006;Newman-Norlund et al., 2007;Williams et al., 2007), the current study is the first to measure the effects of counter-mirror sensorimotor learning on BOLD responses during passive action observation. We investigated the two training groups' responses to action observation by selecting the voxels showing the peak response to the action observation and execution conjunction in the four classical mirror system areas in the Compatible (control) group. This was because the training received by the Compatible group consisted of normal, "mirror" sensorimotor experience; the same as that which all participants would have received during lifelong experience of observing their own actions. 
Comparison between the two groups of responses to action observation in the peak mirror system voxels indicated that, while the Compatible training group showed higher activity in the mirror system when observing hand actions than when observing foot actions, this pattern was reversed in all four areas in the Incompatible training group. Thus, as a result of incompatible sensorimotor learning, voxels that are ordinarily more responsive to the observation of hand actions altered their responses to become more active when observing foot actions. We propose that this is because incompatible training resulted in a population of neurons with novel, counter-mirror properties; these neurons are active during performance of a hand action, but during the observation of a foot action.

It is likely that the Incompatible training group also developed neurons with the complementary type of counter-mirror property, i.e. neurons that are active during the performance of a foot action, but during the observation of a hand action. However, the BOLD response reflects the properties of the more prevalent neurons, and as indicated by our data from the Compatible training group, and by previous studies (e.g. Kollias et al., 2001), mirror areas have a relative "preference" for hand over foot movements. Thus the more prevalent neurons in these mirror areas will be those neurons which are active during the performance of hand, rather than foot, movements. In the Compatible training group these neurons respond more to the observation of hand actions, but following incompatible sensorimotor training they respond more to observation of foot actions. Vogt et al. (2007), in a conjunction analysis for hand actions, revealed z-coordinates in ventral premotor cortex identical to those of the present study. This provides further support for the suggestion that these areas are dominant for the observation and execution of hand movements. Additionally, a withingroup contrast for the Compatible training group, collapsed across the four peak mirror system voxels, showed significantly higher activity during the observation of hand than of foot actions. Premotor cortex dominance for the performance of hand actions over foot actions could be the 
result of the greater need for fine motor control of hand actions, while a sensorimotor account of the development of the mirror system might predict a dominance for the observation of hand over foot actions, because we experience far more self-observation of hand actions than of foot actions.

It is probable that the interactions between training group and observed effector, depicted in Figure 3 , reflect a combination of excitatory and inhibitory learning processes. That is, the data from the Incompatible training group result not only from the formation of new, excitatory associations between the observation of foot and the performance of hand actions, but also from the inhibition of existing associations between the observation and performance of hand actions. A possible way to assess the relative contribution of inhibitory learning is to compare the neural responses to the observation of hand actions between the two groups. Indeed, two of the four peak voxels show a significant difference between the groups during hand action observation. While this result is supportive of an inhibition account, it could also result in part from additional excitatory learning in the Compatible training group (e.g. learning of stimulus-specific features). A sensorimotor hypothesis predicts that both inhibitory and excitatory processes could be involved during learning.

The contrast between the results from the Compatible and Incompatible training groups rules out a number of possible interpretations of these data. During training and testing, subjects in the two groups observed the hand and foot actions, and executed the hand and foot actions, with equal frequency. Therefore, the distinctive pattern of results observed in the Incompatible training group could not have been due solely to unimodal visual experience (Ferrari et al., 2005), to unimodal motor experience, or to the additive effects of these two kinds of unimodal experience (CalvoMerino et al., 2005;Calvo-Merino et al., 2006;Cross et al., 2006). Rather, the additional countermirror activity that occurred in the Incompatible training group must have been due to the 
sensorimotor experience received during training; to the fact that participants in the Incompatible training group had simultaneously seen and performed non-matching, rather than matching, actions during training.

The results of this study confirm the predictions of experience-based theories which postulate that the mirror system consists of links between neural populations coding for sensory and motor action representations, and that these are forged through correlated experience of seeing and doing actions (Heyes, 2001;Keysers and Perrett, 2004;Brass and Heyes, 2005). These theories further suggest that the processes that create these links are stimulus-general associative learning mechanisms. Would the results of the current study have been the same if the stimuli used were more general, e.g. colours or tones, rather than action stimuli? Since these stimuli would not have prior associations with the performance of hand or foot actions, no inhibitory learning would be required, and thus the effects of excitatory learning might even be stronger. Alternatively, it might well be the case that body movement action stimuli have privileged inputs to the mirror system (e.g. via inputs from the superior temporal sulcus; Keysers and Perrett, 2004). If this is the case, only body movement stimuli or those which share salient characteristics with body movements (e.g. robotic movement stimuli; Press et al., 2005;Press et al., 2007) can become associated with the performance of actions within mirror system areas. More general, non-action stimuli might enter into associations with the performance of actions in other areas, such as dorsal premotor cortex (Hoshi and Tanji, 2007). Indeed, during the perception of learned action effect tones, when no response is required, areas such as dorsal premotor cortex and supplementary motor area are active (Elsner et al., 2002; Melcher et al., 2008). 
The experience-based, sensorimotor account of the origins of the mirror system has important implications. The results of this study suggest that there is nothing intrinsically "mirror" about the mirror system. Evolution by natural selection may have prepared certain cortical areas to represent experienced relationships between action-related visual inputs and motor outputs, but it has not specified the nature of these relationships. For example, observation of a hand action does not prime the execution of a hand action by virtue of an innate or intrinsic property of the mirror system. Rather, hand action observation primes hand action execution because, during selfobservation in the course of typical development, the sight of hand actions is typically correlated with performance of hand actions. Sensorimotor experience of the kind required to create mirror neurons is received whenever individuals watch themselves performing an action, directly or in a mirror, and when they are imitated by others or engage in synchronous action with others (Heyes, 2001). Thus the mirror system, thought to play an essential role in our ability to interact with others, may itself acquire its properties as a result of social interactions during development.

Acknowledgements

This work was supported by the European Community's Sixth Framework Programme under contract number: NEST 012929, and by the Economic and Social Research Council (ESRC)'s research centre for Economic Learning and Social Evolution (ELSE). 
Abbreviations

ANOVA, analysis of variance; BA, Brodmann area; BOLD, blood oxygenation level dependent; EMG, electromyogram; EPI, echo planar imaging; fMRI, functional Magnetic Resonance Imaging; GLM, general linear model; MNI, Montreal Neurological Institute; RT, response time; SPM, statistical parametric map; TE, echo time; TR, repetition time.

References

Alegre,M., Lazaro,D., Valencia,M., Iriarte,J. \& Artieda,J. (2006) Imitating versus non-imitating movements: differences in frontal electroencephalographic oscillatory activity. Neurosci Lett., 398, 201-205

Andersson,J.L., Hutton,C., Ashburner,J., Turner,R. \& Friston,K.J. (2001) Modelling geometric deformations in EPI time series. Neuroimage., 13, 903-919.

Arbib,M.A. (2005) From monkey-like action recognition to human language: an evolutionary framework for neurolinguistics. Behav.Brain Sci., 28, 105-124.

Aziz-Zadeh,L., Wilson,S.M., Rizzolatti,G. \& lacoboni,M. (2006) Congruent Embodied Representations for Visually Presented Actions and Linguistic Phrases Describing Actions. Curr.Biol., 16, 1818-1823.

Brass,M., Bekkering,H. \& Prinz,W. (2001a) Movement observation affects movement execution in a simple response task. Acta Psychol.(Amst), 106, 3-22.

Brass,M. \& Heyes,C. (2005) Imitation: is cognitive neuroscience solving the correspondence problem? Trends Cogn Sci., 9, 489-495. 
Brass,M., Zysset,S. \& von Cramon,D.Y. (2001b) The inhibition of imitative response tendencies. Neuroimage., 14, 1416-1423.

Buccino,G., Binkofski,F., Fink,G.R., Fadiga,L., Fogassi,L., Gallese,V., Seitz,R.J., Zilles,K., Rizzolatti,G. \& Freund,H.J. (2001) Action observation activates premotor and parietal areas in a somatotopic manner: an fMRI study. Eur.J.Neurosci., 13, 400-404.

Calvo-Merino,B., Glaser,D.E., Grezes,J., Passingham,R.E. \& Haggard,P. (2005) Action observation and acquired motor skills: an FMRI study with expert dancers. Cereb.Cortex, 15, 1243-1249.

Calvo-Merino,B., Grezes,J., Glaser,D.E., Passingham,R.E. \& Haggard,P. (2006) Seeing or doing? Influence of visual and motor familiarity in action observation. Curr.Biol., 16, 1905-1910.

Catmur,C., Walsh,V. \& Heyes,C. (2007) Sensorimotor learning configures the human mirror system. Curr.Biol., 17, 1527-1531.

Costantini,M., Committeri,G. \& Galati,G. (2008) Effector- and target-independent representation of observed actions: evidence from incidental repetition priming. Exp.Brain Res..

Cross,E.S., Hamilton,A.F. \& Grafton,S.T. (2006) Building a motor simulation de novo: observation of dance by dancers. Neuroimage., 31, 1257-1267.

di Pellegrino,G., Fadiga,L., Fogassi,L., Gallese,V. \& Rizzolatti,G. (1992) Understanding motor events: a neurophysiological study. Exp.Brain Res., 91, 176-180.

Elsner,B., Hommel,B., Mentschel,C., Drzezga,A., Prinz,W., Conrad,B. \& Siebner,H. (2002) Linking actions and their perceivable consequences in the human brain. Neuroimage., 17, 364-372.

Fadiga,L., Fogassi,L., Pavesi,G. \& Rizzolatti,G. (1995) Motor facilitation during action observation: a magnetic stimulation study. J.Neurophysiol., 73, 2608-2611. 
Ferrari,P.F., Rozzi,S. \& Fogassi,L. (2005) Mirror neurons responding to observation of actions made with tools in monkey ventral premotor cortex. J.Cogn Neurosci., 17, 212-226.

Friston,K.J., Holmes,A.P., Worsely,K.J., Poline,J.B., Frith,C.D. \& Frackowiak,R.S.J. (1995) Statistical parametric maps in functional imaging: a general linear approach. Hum.Brain Mapp., 2, 189-210.

Gallese,V. (2007) Before and below 'theory of mind': embodied simulation and the neural correlates of social cognition. Philos.Trans.R.Soc.Lond B Biol.Sci., 362, 659-669.

Gallese,V. \& Goldman,A. (1998) Mirror neurons and the simulation theory of mind-reading. Trends Cogn Sci., 2, 493-501.

Gazzola,V., van der,W.H., Mulder,T., Wicker,B., Rizzolatti,G. \& Keysers,C. (2007) Aplasics born without hands mirror the goal of hand actions with their feet. Curr.Biol., 17, 1235-1240.

Gillmeister,H., Catmur,C., Liepelt,R., Brass,M. \& Heyes,C. (2008) Experience-based priming of body parts: A study of action imitation. Brain Res., 1217, 157-170.

Heyes,C. (2001) Causes and consequences of imitation. Trends Cogn Sci., 5, 253-261.

Heyes,C., Bird,G., Johnson,H. \& Haggard,P. (2005) Experience modulates automatic imitation. Brain Res.Cogn Brain Res., 22, 233-240.

Hommel,B., Musseler,J., Aschersleben,G. \& Prinz,W. (2001) The Theory of Event Coding (TEC): a framework for perception and action planning. Behav.Brain Sci., 24, 849-878.

Hoshi,E. \& Tanji,J. (2007) Distinctions between dorsal and ventral premotor areas: anatomical connectivity and functional properties. Curr.Opin.Neurobiol., 17, 234-242.

lacoboni,M. (2005) Neural mechanisms of imitation. Curr.Opin.Neurobiol., 15, 632-637. 
lacoboni,M., Woods,R.P., Brass,M., Bekkering,H., Mazziotta,J.C. \& Rizzolatti,G. (1999) Cortical mechanisms of human imitation. Science, 286, 2526-2528.

Keysers,C. \& Perrett,D.I. (2004) Demystifying social cognition: a Hebbian perspective. Trends Cogn Sci., 8, 501-507.

Kollias,S.S., Alkadhi,H., Jaermann,T., Crelier,G. \& Hepp-Reymond,M.C. (2001) Identification of multiple nonprimary motor cortical areas with simple movements. Brain Res.Brain Res.Rev., 36, 185195.

Laurienti,P.J., Burdette,J.H. \& Maldjian,J.A. (2003) Separating neural processes using mixed eventrelated and epoch-based fMRI paradigms. J.Neurosci.Methods, 131, 41-50.

Leighton,J. \& Heyes,C. (2008) Hand to mouth: automatic imitation across effector systems. J Exp Psychol Hum Percept Perform.

Melcher,T., Weidema,M., Eenshuistra,R.M., Hommel,B. \& Gruber,O. (2008) The neural substrate of the ideomotor principle: an event-related fMRI analysis. Neuroimage., 39, 1274-1288.

Newman-Norlund,R.D., van Schie,H.T., van Zuijlen,A.M. \& Bekkering,H. (2007) The mirror neuron system is more active during complementary compared with imitative action. Nat.Neurosci., 10, 817818.

Press,C., Bird,G., Flach,R. \& Heyes,C. (2005) Robotic movement elicits automatic imitation. Brain Res.Cogn Brain Res., 25, 632-640.

Press,C., Gillmeister,H. \& Heyes,C. (2007) Sensorimotor experience enhances automatic imitation of robotic action. Proc.Biol.Sci., 274, 2509-2514.

Rizzolatti,G. \& Arbib,M.A. (1998) Language within our grasp. Trends Neurosci., 21, 188-194.

Rizzolatti,G. \& Craighero,L. (2004) The mirror-neuron system. Annu.Rev.Neurosci., 27, 169-192. 
Schubotz,R.I. \& von Cramon,D.Y. (2003) Functional-anatomical concepts of human premotor cortex: evidence from fMRI and PET studies. Neuroimage., 20 Suppl 1:S120-31., S120-S131.

Strafella,A.P. \& Paus,T. (2000) Modulation of cortical excitability during action observation: a transcranial magnetic stimulation study. Neuroreport, 11, 2289-2292.

Sturmer,B., Aschersleben,G. \& Prinz,W. (2000) Correspondence effects with manual gestures and postures: a study of imitation. J.Exp.Psychol.Hum.Percept.Perform., 26, 1746-1759.

Theoret,H. \& Pascual-Leone,A. (2002) Language acquisition: do as you hear. Curr.Biol., 12, R736R737.

Vogt,S., Buccino,G., Wohlschlager,A.M., Canessa,N., Shah,N.J., Zilles,K., Eickhoff,S.B., Freund,H.J., Rizzolatti,G. \& Fink,G.R. (2007) Prefrontal involvement in imitation learning of hand actions: effects of practice and expertise. Neuroimage., 37, 1371-1383.

Wheaton,K.J., Thompson,J.C., Syngeniotis,A., Abbott,D.F. \& Puce,A. (2004) Viewing the motion of human body parts activates different regions of premotor, temporal, and parietal cortex. Neuroimage., 22, 277-288.

Williams,J.H.G., Whiten,A., Waiter,G.D., Pechey,S. \& Perrett,D.I. (2007) Cortical and subcortical mechanisms at the core of imitation. Social Neuroscience, 2, 66-78. 
Table 1. Locations of peak voxels, surviving correction at $p<0.05$ for multiple comparisons across the whole brain, for the conjunction of observation and execution of hand and foot actions in the Compatible training group. Peak mirror system activations are illustrated in Figure 2; superscript indices refer to the labelled activations in Figure 2. Entries in square brackets denote locations of peak mirror system voxels for the conjunction of observation and execution of hand actions alone in the Compatible training group, where these differ from locations for both hand and foot actions.

Premotor cortex (Brodmann area (BA) 6)

Right hemisphere ${ }^{1}$

Left hemisphere ${ }^{2}$

[Left hemisphere

Inferior parietal lobule (BA 40)

Right hemisphere ${ }^{3}$

39

Left hemisphere ${ }^{4}$

[Left hemisphere

\section{Cerebellum}

Right hemisphere

57

$-51$

$-54$

36
0 $-39$

$-45$

$-45$

48

45

$-54$
5.72

$-54$


Left hemisphere

Left superior temporal lobe (BA 48)

Left precuneus (BA 7)

Right superior parietal lobe (BA 7)

Supplementary motor area (BA 32)

Right middle frontal gyrus (BA 6)
5 
Figure Legends

Figure 1. Timelines showing stimuli and procedures during: A. Training, B. Action Observation Task, and C. Action Execution Task. Two trials are depicted for each task. For the training task, the first trial is an example of a compound stimulus trial, while the second is an example of a single stimulus trial.

Figure 2. Rendered images of a reference brain showing "mirror system" areas in the Compatible (control) group. These areas were defined as those showing a significant response to the conjunction of Action Observation and Action Execution, at $p<0.05$, corrected for multiple comparisons across the whole brain. A full list of activations is given in Table 1. Numbers 1 - 4 denote mirror system areas labelled in Table 1.

Figure 3. Parameter estimates of activity in voxels defined by peak responses to the conjunction of Action Observation and Action Execution in each of the four mirror system areas, showing the interaction between observed action (hand, foot) and group (Compatible, Incompatible) during the Action Observation Task. The interaction was significant in all four areas. (A) Left premotor cortex ($51,0,36)$ (MNI coordinates); (B) right premotor cortex $(57,3,36)$; (C) left inferior parietal cortex $(-33,-$ 45,45); (D) right inferior parietal cortex $(39,-39,45)$. Error bars indicate standard error of the mean. 
Figure 1

\section{Training}
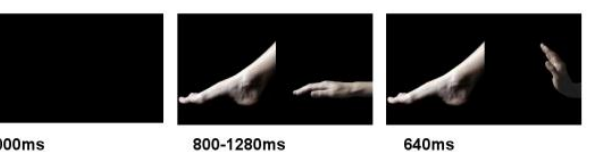

Response:

Compatible: Lift Hand

Incompatible: Lift Foo

800-1280ms

$640 \mathrm{~ms}$

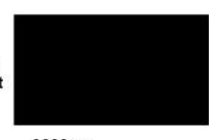

$3000 \mathrm{~ms}$

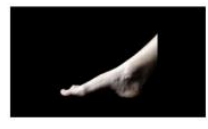

800-1280ms

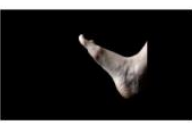

Respons:

Compatible: Lift Foot

Incompatible: Lift Han

$640 \mathrm{~ms}$

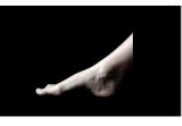

800-1280ms

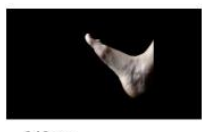

$640 \mathrm{~ms}$

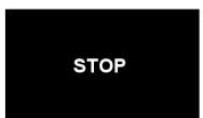

$750 \mathrm{~ms}$

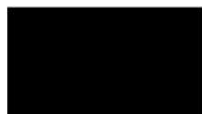

2000-5000ms

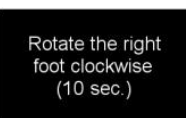

$3000 \mathrm{~ms}$

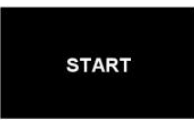

$750 \mathrm{~ms}$

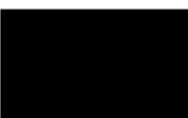

$10000 \mathrm{~ms}$ $750 \mathrm{~ms}$

Figure 2

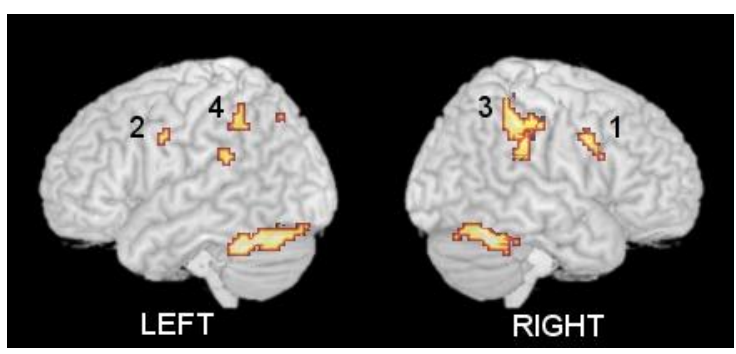

Figure 3 
A
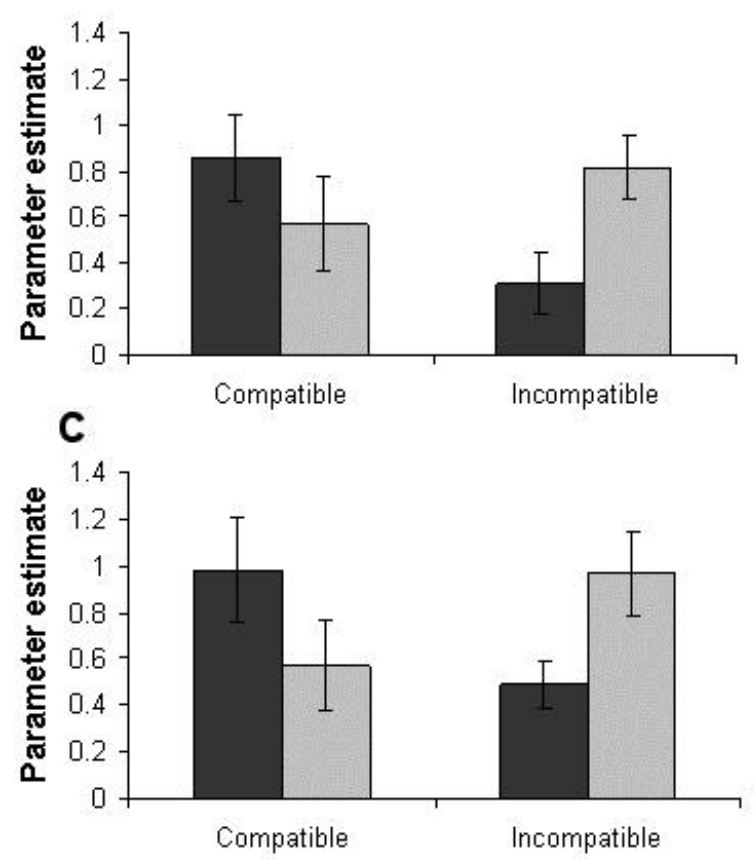

Training group
B
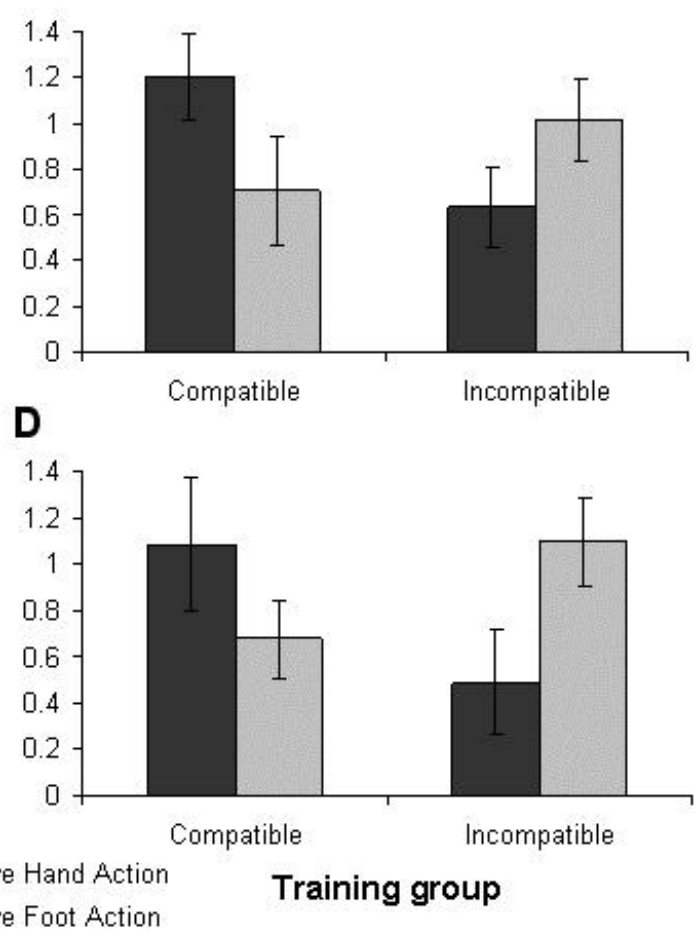